\title{
Uplink Multiuser MIMO Detection Scheme with Reduced Computational Complexity
}

\author{
Soobum $\mathrm{CHO}^{1}$, Sang Kyu PARK ${ }^{2}$ \\ ${ }^{1}$ Dept. of Electrical Engineering, Stanford University, Stanford, CA 94305, USA \\ ${ }^{2}$ Dept. of Electronics and Computer Engineering, Hanyang University, Seoul 133-791, South Korea
}

sbcho98@gmail.com,skpark@hanyang.ac.kr

\begin{abstract}
The wireless communication systems with multiple antennas have recently received significant attention due to their higher capacity and better immunity to fading channels as compared to single antenna systems. A fast antenna selection scheme has been introduced for the uplink multiuser multiple-input multiple-output (MIMO) detection to achieve diversity gains, but the computational complexity of the fast antenna selection scheme in multiuser systems is very high due to repetitive pseudo-inversion computations. In this paper, a new uplink multiuser detection scheme is proposed adopting a switch-and-examine combining (SEC) scheme and the Cholesky decomposition to solve the computational complexity problem. $K$ users are considered that each user is equipped with two transmit antennas for Alamouti space-time block code (STBC) over wireless Rayleigh fading channels. Simulation results show that the computational complexity of the proposed scheme is much lower than the systems with exhaustive and fast antenna selection, while the proposed scheme does not experience the degradations of bit error rate (BER) performances.
\end{abstract}

\section{Keywords}

Cholesky decomposition, fast antenna selection, multiuser MIMO, SEC, STBC

\section{Introduction}

High system capacity, high spectral efficiency, and strong tolerance with respect to interference and multipath propagation are required in wireless communication systems. Multiple-input multiple-output (MIMO) systems satisfy these requirements by increasing the transmission rate and improving the reliability over the fading channel [1-3]. Even though the MIMO systems theoretically have several advantages, it may be difficult to equip over two antennas into mobile devices such as smart phone, tablet PC, and laptop computer. The installation of the multiple antennas into the small devices may lead to high hardware complexity, high cost, large size, and low battery life.
To solve the problem of the MIMO systems, multiuser MIMO approaches have been proposed [4-6]. The multiuser MIMO systems allow multiple users to deploy spatially separated transmit antennas and to deliver independent signal streams. Therefore, multiuser MIMO systems provide higher data rates and need a small number of antennas than the MIMO systems. However, multiuser MIMO channels suffer from co-channel interference (CCI), since they use the same frequency.

An uplink multiuser MIMO detection method for suppressing CCIs was introduced [7]. In this work, CCIs were suppressed using a beamforming process based on the Rayleigh-Ritz theorem, and a number of RF chains were required to achieve sufficient bit error rate (BER) performance. However, since adding a number of RF chains is costly, this is undesirable from a practical point of view. To solve the problem of the beamforming process based on the Rayleigh-Ritz theorem, exhaust and fast antenna selection schemes have been proposed in multiuser detection process [8]. The BER performance of the systems that use such selection schemes is higher than that of systems using the same number of RF chains without any selection. However, in the process of selecting the optimum antenna subset, repetitive matrix inversion computations occur, which is the main cause of the increase in computational complexity. Generally, matrix inversion can be computed via GaussianJordan elimination. However, if the matrix is positive definite, this approach is computationally expensive and does not exploit the characteristics of Hermititan matrices. In this case, it is better to start with the Cholesky decomposition.

Furthermore, a multi-branch switched diversity system based on a fixed switching threshold was introduced [9]. It was also shown that a switch-and-examine combining (SEC) scheme improves performance along the additional diversity branches [10]. In a subset of the available diversity branches with limited resources, the SEC scheme is adequate for selecting branches based on the signal-tointerference plus noise ratio (SINR) with low complexity.

In this paper, the SEC scheme is adopted to multiuser detection in order to reduce the number of repetitive matrix inversion computations. Additionally, the matrices are 
positive definite so the Cholesky decomposition can be used, which has a lower computational complexity than the Gauss-Jordan method.

This paper is organized as follows. The uplink multiuser communication system model is explained in Sec. 2. In Sec. 3, a beamforming process based on the RayleighRitz theorem is performed to suppress CCIs. Section 4 describes proposed multiuser decoding method with reduced computational complexity. Section 5 describes adaptive switching thresholds and the BER according to the fading model. Finally, conclusions are given in Sec. 6 .

\section{System Model}

Figure 1 shows an uplink multiuser MIMO system over a multiple access channel.

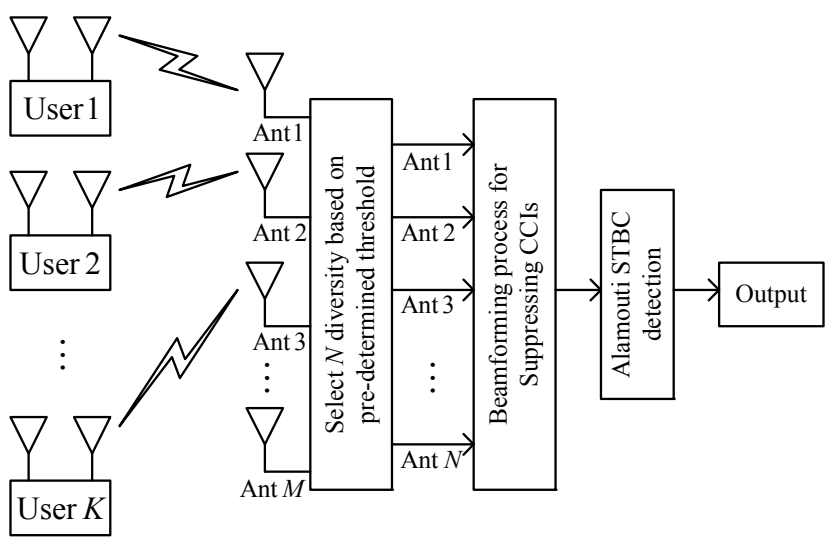

Fig. 1. Uplink multiuser MIMO system based on SEC and beamforming process.

$K$ users are equipped with two transmit antennas, and the base station (BS) has $M$ diversity branches with $N$ available RF chains. It has been proven that when each user has two transmit antennas, using $2 \times(K-1)+r$ receiver antennas for any $r \geq 1$, the receiver can completely separate the signals of the $K$ users, where $r$ is the redundant receiver antenna for obtaining receiver diversity gains [11]. All users use the Alamouti transmission scheme [12] to achieve the transmit diversity gain. During the first time slot, $s_{1}{ }^{(k)}$ and $s_{2}{ }^{(k)}$ are transmitted at two transmit antennas, and during the second time slot, $-\left[s_{2}{ }^{(k)}\right]^{*}$ and $\left[s_{1}{ }^{(k)}\right]^{*}$ are transmitted, where the superscripts $[\cdot]^{*}$ denote complex conjugation and $k=1,2, \ldots, K . s_{1}{ }^{(k)}$ and $s_{2}{ }^{\left({ }^{k}\right)}$ have unit symbol energy. It is assumed that the $K$ users transmit simultaneously in the same frequency band, and their transmissions are perfectly synchronized in time. The channel matrix for user $k$ can be described as

$$
\mathbf{H}^{(k)}=\left[\begin{array}{cc}
h_{11}^{(k)} & h_{12}^{(k)} \\
h_{21}^{(k)} & h_{22}^{(k)} \\
\vdots & \vdots \\
h_{N 1}^{(k)} & h_{N 2}^{(k)}
\end{array}\right]
$$

where $h_{i j}^{(k)}$ is the Rayleigh fading channel gain of user $k$ from the $j$ th transmit antenna to the $i$ th receiver antenna. Each entry in the channel matrix is modeled as a statistically independent and identically distributed (i.i.d.) zeromean complex Gaussian variable with a variance of $1 / 2$. It is assumed that none of the users have channel state information (CSI), whereas CSI is known at the BS.

In reality, the channel between transmit and receive antennas is not ideally the i.i.d. Rayleigh fading because of several factors [13]. One of them is spatial correlation caused by reflection and diffraction wave propagation [14]. If the main scattering appears close to the antenna arrays, the usage of Kronecker model is reasonable [15].

\section{Beamforming Process for CCI Cancellation}

An Alamouti space-time block code (STBC) word sent over the two transmit antennas of user $k$ during two symbol periods is represented as

$$
\mathbf{S}^{(k)}=\left[\begin{array}{cc}
s_{1}^{(k)} & -\left[s_{2}^{(k)}\right]^{*} \\
s_{2}^{(k)} & {\left[s_{1}^{(k)}\right]^{*}}
\end{array}\right], k=1,2, \ldots, K .
$$

The correlation matrix of the Alamouti STBC is expressed as

$$
\mathrm{E}\left[\mathbf{S}^{(k)}\left\{\mathbf{S}^{(k)}\right\}^{H}\right]=2 E_{s} \mathbf{I}_{2}
$$

where $E_{s}$ denotes symbol energy, $\mathbf{I}_{2}$ is a $2 \times 2$ identity matrix, and $E[\cdot]$ is the expected value operator. If user 1 is the desired user, the signal received at the BS can be represented as

$$
\mathbf{Y}=\mathbf{H}^{(1)} \mathbf{S}^{(1)}+\sum_{k=2}^{K} \mathbf{H}^{(k)} \mathbf{S}^{(k)}+\mathbf{n}_{0}
$$

where $\mathbf{n}_{0}$ is an $N \times 2$ matrix that is additive white complex valued Gaussian noise (AWGN), whose elements are i.i.d. Gaussian random variables with zero mean and a variance $\sigma^{2}$.

The main purpose of the beamforming process is to suppress CCIs. In other words, the beamforming process plays a role in minimizing the power of unwanted signals. To suppress CCIs, an $N \times 1$ beamforming weight vector $\mathbf{x}$ for user 1 is assumed and then the received signal $\mathbf{Y}$ is multiplied by $\mathbf{x}^{H}$ at the beamformer. The signal after multiplying $\mathbf{Y}$ by $\mathbf{x}^{H}$ is represented as

$$
\mathbf{x}^{H} \mathbf{Y}=\mathbf{x}^{H} \mathbf{H}^{(1)} \mathbf{S}^{(1)}+\mathbf{x}^{H} \sum_{k=2}^{K} \mathbf{H}^{(k)} \mathbf{S}^{(k)}+\mathbf{x}^{H} \mathbf{n}_{0}
$$

where the superscript $[\cdot]^{H}$ denotes a Hermitian conjugate. The signal correlation matrix $\mathbf{R}_{s}$ is denoted as 


$$
\begin{aligned}
\mathbf{R}_{s} & =E\left[\mathbf{x}^{H} \mathbf{H}^{(1)} \mathbf{S}^{(1)}\left(\mathbf{x}^{H} \mathbf{H}^{(1)} \mathbf{S}^{(1)}\right)^{H}\right] \\
& =2 E_{s} \mathbf{x}^{H} \mathbf{H}^{(1)}\left\{\mathbf{H}^{(1)}\right\}^{H} \mathbf{x}
\end{aligned}
$$

and the interference plus noise correlation matrix $\mathbf{R}_{\text {in }}$ is expressed as

$$
\begin{aligned}
\mathbf{R}_{\text {in }} & =E\left[\left(\mathbf{x}^{H} \sum_{k=2}^{K} \mathbf{H}^{(k)} \mathbf{S}^{(k)}+\mathbf{x}^{H} \mathbf{n}_{0}\right)\left(\mathbf{x}^{H} \sum_{k=2}^{K} \mathbf{H}^{(k)} \mathbf{S}^{(k)}+\mathbf{x}^{H} \mathbf{n}_{0}\right)^{H}\right] \\
& =\mathbf{x}^{H}\left[\sum_{k=2}^{K} \mathbf{H}^{(k)} E\left[\mathbf{S}^{(k)}\left\{\mathbf{S}^{(k)}\right\}^{H}\right]\left\{\mathbf{H}^{(k)}\right\}^{H}+E\left[\mathbf{n}_{0}\left\{\mathbf{n}_{0}\right\}^{H}\right]\right] \mathbf{x} \\
& =\mathbf{x}^{H}\left(E_{s} \sum_{k=2}^{K} \mathbf{H}^{(k)} 2 E_{s} \mathbf{I}_{2}\left\{\mathbf{H}^{(k)}\right\}^{H}+2 \sigma^{2} \mathbf{I}_{M}\right) \mathbf{x}
\end{aligned}
$$

where it is assumed that $E_{S}$ is unit symbol energy. Finally, the output SINR of the desired user 1 at the beamformer is defined as

$$
\operatorname{SINR}^{(1)}=\frac{\mathbf{R}_{s}}{\mathbf{R}_{i n}}=\frac{\mathbf{x}^{H} \mathbf{H}^{(1)}\left\{\mathbf{H}^{(1)}\right\}^{H} \mathbf{x}}{\mathbf{x}^{H}\left[\sum_{k=2}^{K} \mathbf{H}^{(K)}\left\{\mathbf{H}^{(K)}\right\}^{H}+\sigma^{2} \mathbf{I}_{M}\right] \mathbf{x}} .
$$

The goal of beamforming process is to find the beamforming weight vector $\mathbf{x}$ that maximizes the output SINR of user 1. From (8), the problem of maximizing the output SINR can be considered as a special case of the more general problem of finding a critical point of the Rayleigh quotient, and its value is bounded by the maximum and minimum eigenvalues of $\left[\sum_{k=2}^{K} \mathbf{H}^{(k)}\left\{\mathbf{H}^{(k)}\right\}^{H}+\sigma^{2} \mathbf{I}_{M}\right]^{-1} \mathbf{H}^{(1)}\left\{\mathbf{H}^{(1)}\right\}^{H}$. To maximize the output SINR, $\mathbf{x}$ should be chosen as the eigenvector of $\left[\sum_{k=2}^{K} \mathbf{H}^{(k)}\left\{\mathbf{H}^{(k)}\right\}^{H}+\sigma^{2} \mathbf{I}_{M}\right]^{-1} \mathbf{H}^{(1)}\left\{\mathbf{H}^{(1)}\right\}^{H}$ associated with the maximum eigenvalue. By multiplying $\mathbf{x}^{H}$ by the received signal $\mathbf{Y}$, a $1 \times 2$ equivalent channel vector is obtained, which seems like a channel matrix when it is equipped with two transmit antennas and one receiver antenna. It enables to detect user 1 by using a general Alamouti decoding scheme.

\section{Multiuser Detection with Reduced Computational Complexity}

Fast antenna selection schemes improve system performance with a low hardware cost. If the BS has $M$ diversity branches with $N$ available RF chains, there are $C_{N}^{M}$ possible antenna subsets, which are expressed as

$$
\Theta=\left\{\Phi_{1}, \Phi_{2}, \ldots, \Phi_{C_{N}^{M}}\right\}
$$

where $C_{N}^{M}$ denotes the number of ways to choose $N$ elements from a set of $M$ elements and $\Phi_{i}$ denotes the $i$ th antenna subset. In multiuser MIMO systems, the system performance is mainly determined by the minimum SINR among all users. Therefore, if $\mathrm{SINR}_{\min , i}$ denotes the minimum SINR among all users for the subset $\Phi_{i}$, the best antenna subset can be selected by using a max-min criterion, represented as

$$
\Phi_{\text {opt }}=\arg \max _{\Phi_{i} \in \Theta} \operatorname{SINR}_{\min , i}, i=1,2, \ldots, C_{N}^{M} .
$$

To search for the optimum antenna subset that satisfies (10), repetitive SINR computations are required between each user and the BS equipped with subset $\Phi_{i}$. If there are $C_{N}^{M}$ antenna subsets, $K \times C_{N}^{M}$ SINR computations are required for selecting the best antenna subset. Moreover, in the SINR calculation process, the matrix inversion of $\left[\sum_{k=2}^{K} \mathbf{H}^{(k)}\left\{\mathbf{H}^{(k)}\right\}^{H}+\sigma^{2} \mathbf{I}_{M}\right]$ should be calculated, which makes the computational complexity high. In short, the complexity of the fast antenna selection scheme in multiuser systems is very high due to the many pseudo-inverse computations.

In this paper, two alternative solutions are proposed based on the SEC scheme and the Cholesky decomposition to solve the problem mentioned above and to compare the systems in terms of computational complexity and BER performance. The SEC scheme is adopted in multiuser detection in order to reduce the number of repetitive matrix inversions. Additionally, since the matrix is positive definite, the Cholesky decomposition can be exploited, which is less computationally complex than the GaussJordan method.

\subsection{Switch-and-Examine Combining}

In the case of a conventional SEC in a single user system, the receiver needs to monitor the signal quality of the currently used diversity branch and to compare it with the pre-determined switching threshold. If the signal quality falls below the threshold, the receiver switches to another diversity branch.

In this paper, the SEC scheme is adopted to the multiuser detection system instead of fast antenna selection schemes in order to reduce the number of repetitive matrix inversions while maintaining BER performance. In the proposed multiuser detection method, the receiver first calculates the switching decision criterion presented as the square of the Frobenius norm of the minimum SINR matrix from the currently in use antenna subset $\Phi_{i}$ and then compares it with the pre-determined switching threshold. If the switching decision criterion falls below the pre-determined threshold, the receiver switches to another antenna subset. For the SEC, special care must be taken in choosing the switching threshold for performance maximization, and the proper thresholds that improve BER performance can be obtained through repetitive simulations. The detailed algorithm is depicted in Fig. 2. 


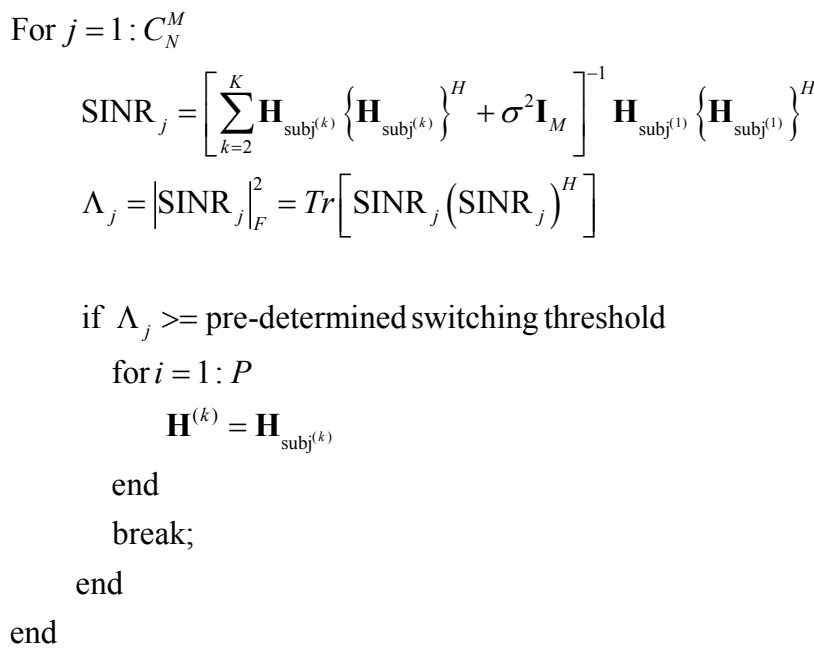

Fig. 2. Algorithm for the proper threshold.

In the algorithm, $\mathbf{H}_{\text {subj }^{(k)}}$ denotes the channel matrix between user $k$ and the antenna subset $\Phi_{i}$. If the minimum SINRs from all possible antenna subsets are smaller than the pre-determined switching threshold, the best antenna subset is selected by using the max-min criterion.

\subsection{Cholesky Decomposition}

Generally, Cholesky decomposition is used to solve linear equations for which the coefficient matrix is a special form, namely, positive definite. A Hermitian matrix is positive definite if it satisfies

$$
\mathbf{a}^{H} \mathbf{A} \mathbf{a}>0,
$$

where a denotes all nonzero vectors. If $\mathbf{A}$ is positive definite, it can be decomposed in exactly one way into the following form:

$$
\mathbf{A}=\mathbf{R}^{H} \mathbf{R},
$$

where $\mathbf{R}$ is upper triangular, and all main diagonal entries are positive. $\mathbf{R}$ is called the Cholesky factor of $\mathbf{A}$. As it is already known, the matrix inversion of $\left[\sum_{k=2}^{K} \mathbf{H}^{(k)}\left\{\mathbf{H}^{(k)}\right\}^{H}+\sigma^{2} \mathbf{I}_{M}\right]$ should be calculated repetitively to search for the optimum antenna subset. One thing to note is that the matrix $\left[\sum_{k=2}^{K} \mathbf{H}^{(k)}\left\{\mathbf{H}^{(k)}\right\}^{H}+\sigma^{2} \mathbf{I}_{M}\right]$ is positive definite, so it is more efficient to start with the Cholesky decomposition and then invert the lower triangular matrix and compute its Gram. First, it is assumed that $\mathbf{A}=\left[\sum_{k=2}^{K} \mathbf{H}^{(k)}\left\{\mathbf{H}^{(k)}\right\}^{H}+\sigma^{2} \mathbf{I}_{M}\right]$ and $\mathbf{A}$ is decomposed as $\mathbf{A}=\mathbf{R}^{H} \mathbf{R}$ using Cholesky's method. The lower triangular matrix $\mathbf{R}$ can be inverted, and then the $\operatorname{Gram}\left(\mathbf{R}^{-1}\right)^{\mathrm{H}} \mathbf{R}^{-1}$ of $\mathbf{R}^{-1}$ can be built. As a result, $\frac{1}{2} N^{3}+\frac{3}{2} N^{2}$ multiplications, $\frac{1}{2} N^{3}-\frac{1}{2} N^{2}$ summations, and $N$ square-root operations are required to calculate the inverse of $\mathbf{A}$, which yields the FLOPs of Cholesky's method as

$$
\text { FLOPs of Cholesky's method }=N^{3}+N^{2}+N \text {. }
$$

In contrast, the Gauss-Jordan elimination method is used to find inverse matrices in normal cases. In this case, the routine solves the $N$ versions of the linear equation problem with different constant vectors. Therefore, the FLOPs for the Gauss-Jordan method is denoted as

$$
\text { FLOPs of the Gauss-Jordan method }=\frac{1}{2} N^{4}+\frac{3}{2} N^{3} .
$$

However, this method is computationally expensive and does not exploit the Hermitian structure of the matrix. In short, when matrix $\mathbf{A}$ is positive definite, it is more efficient to calculate its inverse with the Cholesky decomposition rather than Gauss-Jordan elimination.

\section{Simulation Results}

In this section, simulation results are provided that confirm the performance of the system model explained in the previous sections. The BER performance comparisons of various multiuser detection methods are shown over a Rayleigh fading channel. It is assumed that two and three users are equipped with two antennas, and each user transmits an Alamouti STBC. Quadrature phase shift keying (QPSK) modulation scheme is used, which provides a rate equal to two bits per channel user. To effectively suppress CCIs, more than $2 \times(K-1)+r$ receiver antennas should be selected. In addition, the fast antenna selection and SEC schemes are used in the detection process to achieve receiver diversity gains. The threshold values for the simulation of the proposed multiuser detection method are given in Tab. 1. When the number of user $K$ is 2 and the number of available RF chain $N$ is 3 , it is assumed that the number of diversity branch $M$ is 4 or 5 . Furthermore, it is assumed that $M$ is 6 or 7 , when $K$ is 3 and $N$ is 5 .

Fig. 3 and Fig. 4 show the comparisons of BER performances of the proposed multiuser detection method, fast antenna selection scheme, and no selection scheme, when the number of user is two and three respectively. Fig. 3 and Fig. 4 demonstrate that the system with the fast antenna selection and the proposed multiuser detection method

\begin{tabular}{|c|c|c|c|c|}
\hline \multirow{2}{*}{$E_{\mathrm{b}} / N_{0}(\mathrm{~dB})$} & \multicolumn{4}{|c|}{ Proper threshold } \\
\cline { 2 - 5 } & \multicolumn{2}{|c|}{$K=2$} & \multicolumn{2}{c|}{$K=3$} \\
\cline { 2 - 5 } & $M=4, N=3$ & $M=5, N=3$ & $M=6, N=5$ & $M=7, N=5$ \\
\hline 0 & 1.7 & 3 & 1.7 & 1.4 \\
\hline 2.5 & 6 & 10 & 9 & 9 \\
\hline 5 & 22 & 30 & 3.5 & 35 \\
\hline 7.5 & 42 & 80 & 80 & 80 \\
\hline 10 & 120 & 180 & 160 & 250 \\
\hline 12.5 & 240 & 400 & 310 & 400 \\
\hline 15 & 500 & 900 & 500 & 980 \\
\hline
\end{tabular}

Tab. 1. Optimum threshold values for the proposed multiuser detection method. 


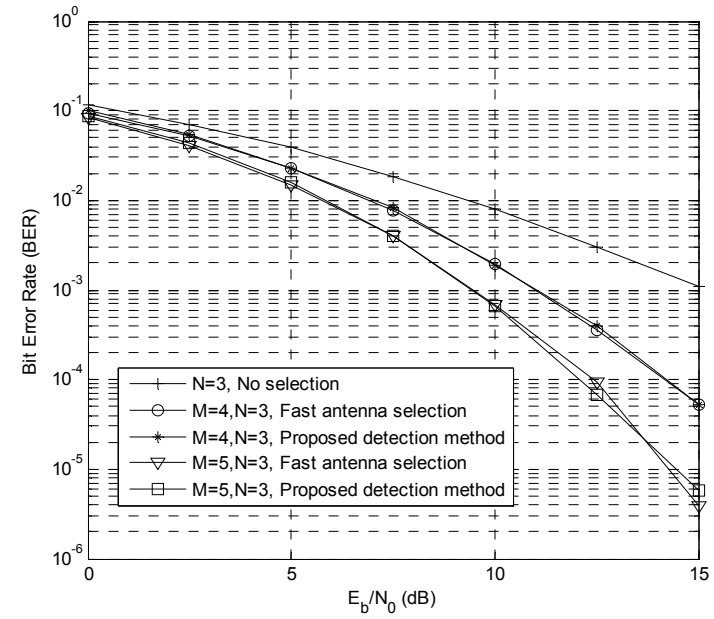

Fig. 3. Comparison of BER performance with two users.

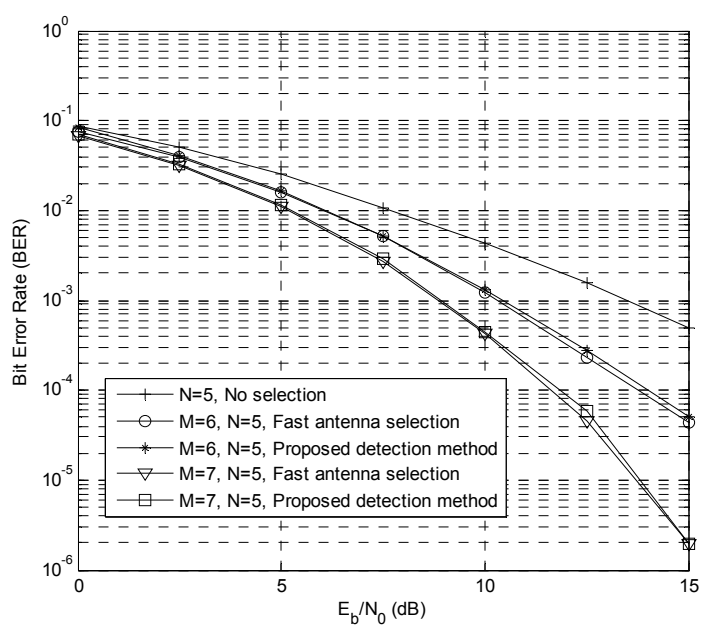

Fig. 4. Comparison of BER performance with three users.

outperforms the system without the selection schemes in terms of BER performance. The existence of diversity gains can be recognized by confirming the slope of the BER curves between the system with the selection scheme and the system without it. Moreover, Fig. 3 and Fig. 4 also show that the BER performance of the system with the proposed multiuser detection method is very close to that of the fast antenna selection scheme. Therefore, it is clear that the proposed multiuser detection method can significantly reduce the FLOPs without the degradation of the BER performance.

As already mentioned in Sec. 4, in the process of searching for the optimal receiver antenna subset, the repetitive matrix inversion computations required to obtain the minimum SINR for each possible subset are the main cause of the high computational complexity. Therefore, reducing the number of computations in the matrix inversion can be a valuable method for lowering system complexity.

When BS has three RF chains, Fig. 5 and Fig. 6 represent the number of computations of matrix inversion that occur repetitively until final optimum antenna subset is selected. The results prove that the computational complexity of the proposed multiuser detection method is much

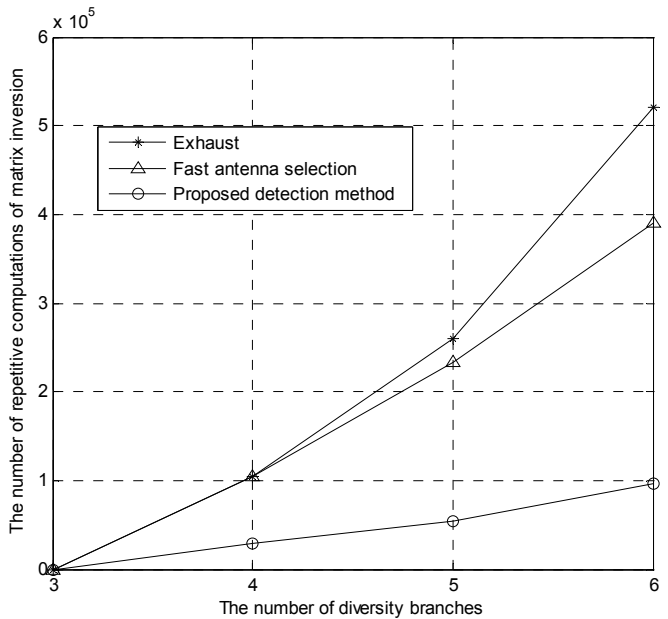

Fig. 5. Comparison of the number of repetitive computations of matrix inversion as a function of the number of available diversity branches with two users.

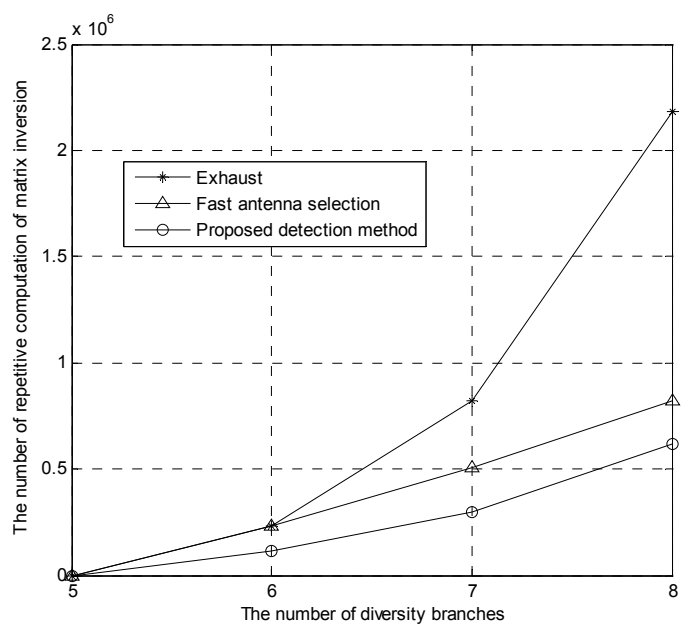

Fig. 6. Comparison of the number of repetitive computations of matrix inversion as a function of the number of available diversity branches with three users.

lower than that of the systems with exhaustive and fast antenna selection. It is evident that the system adopting the fast antenna selection scheme is poor in terms of complexity performance, since it is efficient when $M \gg>N$. Therefore, if the number of available diversity branches $M$ increases, the fast antenna selection scheme makes great performance difference as compared with exhaustive selection, whereas the proposed multiuser detection method still has good performance despite $M \gg>N$. Therefore, the proposed multiuser detection method has the best performance from a computational complexity point of view regardless of the difference between $M$ and $N$.

We also compare the proposed method with other Exhaust and Fast antenna selection methods by means of computational time. To compare the time, we assume that the system uses $2.5 \mathrm{GHz} \mathrm{CPU}$ and one command occurs in each clock time. We also assume that $\mathrm{BS}$ has three RF chains and the numbers of diversity branches are 3, 4, 5, and 6 for two users and 5,6, 7, and 8 for three users. Tab. 2 and Tab. 3 show the comparison of the time when the number of users is two and three respectively. 


\begin{tabular}{|c|c|c|c|}
\hline $\begin{array}{c}\text { The number of } \\
\text { diversity } \\
\text { branches }\end{array}$ & Exhaust & $\begin{array}{c}\text { Fast antenna } \\
\text { selection }\end{array}$ & $\begin{array}{c}\text { Proposed } \\
\text { method }\end{array}$ \\
\hline 4 & $40 \mu \mathrm{s}$ & $40 \mu \mathrm{s}$ & $12 \mu \mathrm{s}$ \\
\hline 5 & $104 \mu \mathrm{s}$ & $94 \mu \mathrm{s}$ & $22 \mu \mathrm{s}$ \\
\hline 6 & $208 \mu \mathrm{s}$ & $156 \mu \mathrm{s}$ & $40 \mu \mathrm{s}$ \\
\hline
\end{tabular}

Tab. 2. Comparison of the computational time as a function of the number of available diversity branches with 2 users.

\begin{tabular}{|c|c|c|c|}
\hline $\begin{array}{c}\text { The number of } \\
\text { diversity } \\
\text { branches }\end{array}$ & Exhaust & $\begin{array}{c}\text { Fast antenna } \\
\text { selection }\end{array}$ & $\begin{array}{c}\text { Proposed } \\
\text { method }\end{array}$ \\
\hline 6 & $0.1 \mathrm{~ms}$ & $0.1 \mathrm{~ms}$ & $0.052 \mathrm{~ms}$ \\
\hline 7 & $0.32 \mathrm{~ms}$ & $0.2 \mathrm{~ms}$ & $0.12 \mathrm{~ms}$ \\
\hline 8 & $0.88 \mathrm{~ms}$ & $0.28 \mathrm{~ms}$ & $0.25 \mathrm{~ms}$ \\
\hline
\end{tabular}

Tab. 3. Comparison of the computational time as a function of the number of available diversity branches with 3 users.

\section{Conclusion}

In this paper, an uplink multiuser MIMO detection scheme using the SEC and Cholesky decomposition was proposed for reducing system complexity performance. The proposed multiuser detection method was compared with the system adopting exhaustive and fast antenna selection with regard to BER and computational complexity over a Rayleigh fading channel employing QPSK modulation. In addition, the differences in the FLOP counts and computational time are compared for the matrix inversion calculation between the Cholesky decomposition and Gauss-Jordan elimination. As a result, it was shown that the three detection schemes have similar BER performance, whereas the proposed multiuser detection method has a much lower computational complexity when the difference between $M$ and $N$ is small. Furthermore, the FLOP counts and computational time for matrix inversion based on the Cholesky decomposition was much lower than those for Gauss-Jordan elimination.

For future work, we will analyze the effects between antenna arrays of point sources and virtual point sources to reduce the system computational complexity for real world environment. In this paper, we assumed that each user is equipped with two antennas. Therefore, we will extend our results when each user uses more antennas which cause degradations of the robustness because of the antenna characteristics such as the gain, the nonuniform distribution of the emitted power, the distance and the coupling between antennas. We will also apply the effect of channel correlation.

\section{References}

[1] GOLDSMITH, A. Wireless Communications. Cambridge: Cambridge University Press, 2005
[2] WU, Q., YANG, X.-S., ZHANG, Y.-T. Research on $2 \times 2$ MIMO channel with truncated Laplacian azimuth power spectrum. Radioengineering, 2013, vol. 22, no. 2, p. 544-548.

[3] DIOUM, I., CLEMENTE, M., DIALLO, A., LUXEY, C., ROSSI, J. P., FARSSI, S. M. Meandered monopoles for $700 \mathrm{MHz}$ LTE handsets and improved MIMO channel capacity performance. Radioengineering, 2011, vol. 20, no. 4, p. 726-732.

[4] LIM, C., YOO, T., CLERCKX, B., LEE, B., SHIM, B. Recent trend of multiuser MIMO in LTE-advanced. IEEE Communications Magazine, 2013, vol. 51, no. 3, p. 127-135. DOI: 10.1109/MCOM.2013.6476877

[5] PHROMPICHAI, S. SGD frequency-domain space-frequency semiblind multiuser receiver with an adaptive optimal mixing parameter. Radioengineering, 2013, vol. 22, no. 1, p. 400-410.

[6] CZINK, N., BANDEMER, B., OESTGES, C., ZEMEN, T., PAULRAJ, A. Analytical multi-user MIMO channel modeling: subspace alignment matters. IEEE Transactions on Wireless Communications, 2012, vol. 11, no. 1, p. 367-377. DOI: 10.1109/TWC.2012.010412.110980

[7] SUN, C., KARMAKAR, N. C., LIM, K. S., FENG, A. Combining beamforming with Alamouti scheme for multiuser MIMO communications. In Proceedings of the $60^{\text {th }}$ IEEE Vehicular Technology Conference (VTC 2004 Fall). Los Angeles (CA, USA), 2004, vol. 2, p. 1415-1419. DOI: 10.1109/VETECF.2004.1400257

[8] CHEN, M.-X., XU, C.-Q., ZHANG, X.-G. Fast antenna selection for Alamouti multi-user MIMO detection. In Proceedings of the $4^{\text {th }}$ International Conference on Wireless Communications, Networking and Mobile Computing. Dalian (China), 2008, p. 1-4. DOI: 10.1109/WiCom.2008.112

[9] YANG, H.-C., ALOUINI, M.-S. Performance analysis of multibranch switched diversity systems. IEEE Transactions on Communications, 2003, vol. 51, no. 5, p. 782-794. DOI: 10.1109/TCOMM.2003.811408

[10] NAM, H., ALOUINI, M.-S. Multi-branch switched diversity with adaptive switching thresholds. In Proceedings of the International Symposium on Information Theory and its Applications. Auckland (New Zealand), 2008, p. 1-6.

[11] TAROKH, V., NAGUIB, A., SESHADRI, N., CALDERBANK, A R. Combined array processing and space-time coding. IEEE Transactions on Information Theory, 1999, vol. 45, no. 4, p. 1121 to 1128. DOI: $10.1109 / 18.761255$

[12] ALAMOUTI, S. M. A simple transmit diversity technique for wireless communications. IEEE Journal on Selected Areas in Communications, 1998, vol. 16, no. 8, p. 1451-1458. DOI: $10.1109 / 49.730453$

[13] GESBERT, D., BOLCSKEI, H., GORE, D. A., PAULRAJ, A. J. Outdoor MIMO wireless channels: models and performance prediction. IEEE Transactions on Communications, 2002, vol. 50, no. 12, p. 1926-1934. DOI: 10.1109/TCOMM.2002.806555

[14] LOYKA, S. L. Channel capacity of MIMO architecture using the exponential correlation matrix. IEEE Communications Letters, 2001, vol. 5, no. 9, p. 369-371. DOI: 10.1109/4234.951380

[15] YU, K., BENGTSSON, M., OTTERSTEN, B., MCNAMARA, D., KARLSSON, P., BEACH, M. Modeling of wide-band MIMO radio channels based on NLoS indoor measurements. IEEE Transactions on Vehicular Technology, 2004, vol. 53, no. 3, p. 655-665. DOI: 10.1109/TVT.2004.827164

\section{About the Authors ...}

Soobum CHO received the B.S. degree in Electronics Engineering from Dong-A University, Korea, in 2006, the 
M.S. and Ph.D. degrees in Electronics and Computer Engineering from Hanyang University, Korea, in 2008 and 2012, respectively. He is currently a postdoctoral visiting scholar in Electrical Engineering at Stanford University, California. His research interests are in the areas of wireless communications theory, massive MIMO, wireless power transfer, and car connectivity technique.

Sang Kyu PARK received the B.S. degree from Seoul National University, Korea, in 1974, the M.S. degree from Duke University, U.S.A. in 1980, and the Ph.D. degree from the University of Michigan, U.S.A. in 1987, all in
Electrical Engineering. From July 1976 to October 1978, he was a research Engineer at the Agency for Defense Development, Korea. From August 1990 to August 1991, he was a visiting scholar at the University of Southern California, U.S.A. Since March 1987, he has been with the Department of Electronics and Computer Engineering at Hanyang University, Korea, where he is currently a Professor. His research interests are in the areas of communications theory, wireless communications, mobile communications, spread spectrum communications, and secure communications. 